\title{
(6) OPEN ACCESS \\ Relationship between macular pigment and visual function in subjects with early age-related macular degeneration
}

\author{
Kwadwo Owusu Akuffo, ${ }^{1}$ John M Nolan, ${ }^{1}$ Tunde Peto, ${ }^{2}$ Jim Stack, ${ }^{1}$ Irene Leung, ${ }^{2}$ \\ Laura Corcoran, ${ }^{1}$ Stephen Beatty ${ }^{1}$
}

\begin{abstract}
${ }^{1}$ Nutrition Research Centre Ireland, Macular Pigment Research Group, School of Health Sciences, Waterford Institute of Technology, Waterford, Ireland ${ }^{2}$ NIHR Biomedical Research Centre at Moorfields Eye Hospital NHS Foundation Trust and UCL Institute of Ophthalmology, London, UK
\end{abstract}

Correspondence to Dr Kwadwo Owusu Akuffo, Macular Pigment Research Group, Vision Research Centre, Waterford Institute of Technology, West Campus, Carriganore, Waterford X91 K236, Ireland; kakuffo@wit.ie

Received 20 January 2016 Revised 11 March 2016 Accepted 15 March 2016 Published Online First 18 April 2016

\section{CrossMark}

\section{To cite: Akuffo KO,}

Nolan JM, Peto T, et al. $\mathrm{Br} J$

Ophthalmol 2017;101:190-

197.

\begin{abstract}
Purpose To investigate the relationship between macular pigment (MP) and visual function in subjects with early age-related macular degeneration (AMD).

Methods 121 subjects with early AMD enrolled as part of the Central Retinal Enrichment Supplementation Trial (CREST; ISRCTN13894787) were assessed using a range of psychophysical measures of visual function, including best corrected visual acuity (BCVA), letter contrast sensitivity (CS), mesopic and photopic CS, mesopic and photopic glare disability (GD), photostress recovery time (PRT), reading performance and subjective visual function, using the National Eye Institute Visual Function Questionnaire-25 (NEI VFQ-25). MP was measured using customised heterochromatic flicker photometry.

Results Letter CS, mesopic and photopic CS, photopic $G D$ and mean reading speed were each significantly $(p<0.05)$ associated with MP across a range of retinal eccentricities, and these statistically significant relationships persisted after controlling for age, sex and cataract grade. BCVA, NEI VFQ-25 score, PRT and mesopic GD were unrelated to MP after controlling for age, sex and cataract grade ( $p>0.05$, for all).

Conclusions MP relates positively to many measures of visual function in unsupplemented subjects with early AMD. The CREST trial will investigate whether enrichment of MP influences visual function among those afflicted with this condition.

Trial registration number ISRCTN13894787.
\end{abstract}

\section{INTRODUCTION}

The carotenoids (lutein (L), zeaxanthin $(\mathrm{Z})$ and meso-zeaxanthin $(\mathrm{MZ})$ ) are found in the human macula, where they are collectively known as macular pigment (MP). MP gives the macula its eponymous yellow appearance (macula lutea). Interestingly, the pigment is captured and normally (typically) distributed at the macula in such a way that it peaks centrally at the foveola (the part of the retina responsible for high acuity and colour vision) and declines with increasing retinal eccentricity.

MP filters short-wavelength light (thereby passively limiting photo-oxidative damage), and its constituent carotenoids have antioxidant properties (and therefore actively neutralise reactive oxygen species). ${ }^{2}$

Several hypotheses (eg, the acuity hypothesis, ${ }^{3}$ glare hypothesis, ${ }^{4}$ visibility hypothesis ${ }^{5}$ ) have been put forward to explain how MP may influence visual function. Indeed, studies have been performed to examine the role of MP for visual function across diverse populations of subjects (eg, healthy subjects free of retinal disease and subjects with age-related macular degeneration (AMD)). In general, cross-sectional studies have shown positive and statistically significant relationships between MP and visual function. ${ }^{4}$ 6-10 Furthermore, most interventional trials have shown that supplementation with the macular carotenoids impacts positively on visual function in subjects with and without retinal disease, ${ }^{11-16}$ and it appears that supplementation with all three macular carotenoids offers advantages over formulations containing only two of these three nutrients. ${ }^{15} 16$ However, we await the outcome of a double-blind placebo-controlled clinical trial to confirm this hypothesis. ${ }^{17}$

The Central Retinal Enrichment Supplementation Trials (CREST), which commenced in 2012 and will be completed in 2016, was designed to investigate the impact of macular carotenoid supplementation on vision in normal healthy subjects with low MP (Trial 1: CREST Normal (ISRCTN68270512)) and subjects with early AMD (the latter representing the study population in the current investigation; Trial 2: CREST AMD (ISRCTN13894787)). ${ }^{17}$ In this report, we present findings on the relationship between MP and psychophysical (and subjective) measures of visual function in unsupplemented subjects with early AMD, using baseline data from CREST AMD. ${ }^{17}$

\section{METHODS}

\section{Study population}

The design and methodology of CREST AMD have been described in detail elsewhere. ${ }^{17}$ Inclusion criteria included: early AMD (one to eight on AREDS 11 -step severity scale ${ }^{18}$ in at least one eye (the study eye), confirmed by the Reading Centre at the Moorfields Eye Hospital Reading Centre, London, UK); best corrected visual acuity (BCVA) of $6 / 12$ or better; spherical equivalence of refraction no more than 5 dioptres; no previous consumption of supplements containing the macular carotenoids $(\mathrm{L}, \mathrm{Z}$ and/or MZ); no retinal pathology beyond AMD; no diabetes mellitus (by self-report). All clinical assessments were conducted by the study investigator (KOA) who was trained in all aspects of the CREST protocol. ${ }^{17}$ Clinical assessments pertaining to this report are briefly described below.

\section{MP measurement}

MP was measured using the Macular Densitometer (Macular Metrics, Providence, Rhode Island, USA) 
at $0.25^{\circ}, 0.5^{\circ}, 1.0^{\circ}$ and $1.75^{\circ}$ retinal eccentricity, with a reference point at $7^{\circ}$. This protocol has been validated for subjects with early AMD. ${ }^{19}$

\section{Visual function assessment}

Best corrected visual acuity

BCVA was measured using the Early Treatment Diabetic Retinopathy Study (ETDRS) logarithm of the minimum angle of resolution (LogMAR) chart (Test Chart 2000 Xpert; Thomson Software Solutions, UK) viewed at $4 \mathrm{~m}$.

\section{Letter contrast sensitivity}

Letter contrast sensitivity (CS) was assessed using the LogMAR ETDRS (Test Chart 2000 PRO; Thomson Software Solutions, UK) chart viewed at $4 \mathrm{~m}$.

\section{CS with the Functional Vision Analyzer}

CS was also assessed using the Functional Vision Analyzer (Stereo Optical Co., Chicago, Illinois, USA). The test was conducted under four simulated conditions: (1) mesopic (3.0 candela per metre square $\left.\left(\mathrm{cd} / \mathrm{m}^{2}\right)\right),(2)$ photopic $\left(85 \mathrm{~cd} / \mathrm{m}^{2}\right),(3)$ mesopic with glare (28 Lux, mesopic glare disability (GD)), (4) photopic with glare (135 Lux, photopic GD).

\section{Photostress recovery time}

Photostress recovery time (PRT) (in seconds) was assessed by measuring the time of recovery (time taken to see the 6/24 letterset ETDRS (Test Chart 2000 PRO) at a specified contrast threshold) after exposure to a $300 \mathrm{~W}$ tungsten spotlight (ARRI 300 Plus lamp, ARRI Lighting Solutions, GmbH, Germany) with a low-pass glass dichroic filter.

\section{Reading performance}

Reading performance was assessed using the English version of the Radner reading chart at $40 \mathrm{~cm}$.

\section{Subjective visual function}

Subjective visual function was assessed using the National Eye Institute Visual Function questionnaire-25 (NEI VFQ-25).

\section{Cataract grading}

Cataract grading was performed using the Haag-Streit BM 900 Slit lamp biomicroscope (Haag-Streit AG, Switzerland) adhering to the Lens Opacities Classification System III (LOCS III) ${ }^{20}$ within the first year of this study by a trained and certified grader (KOA).

\section{Statistical analysis}

One eye (the study eye) of each subject comprised the unit of analysis. Statistical analysis was performed using IBM SPSS Statistics for Windows, V.21.0 (Armonk, New York, USA). Pearson's correlation coefficients were used to investigate bivariate relationships between MP and visual function parameters. General linear models were used to control for variables such as age, sex and cataract grade (while excluding subjects with pseudophakia). We used the 5\% level of significance throughout, without adjusting for multiple comparisons.

\section{RESULTS}

Table 1 presents the demographic and lifestyle characteristics of all subjects included in this report. Table 2 presents the relationship between MP and visual function, as assessed by the bivariate Pearson correlation coefficients. General linear models were used to assess which visual function variables are statistically
Table 1 Demographic, lifestyle, visual function and MP of subjects included in this report

\begin{tabular}{|c|c|}
\hline Variables & n (\%) \\
\hline \multicolumn{2}{|l|}{ Sex } \\
\hline Male & $40(33.1)$ \\
\hline Female & $81(66.9)$ \\
\hline \multicolumn{2}{|l|}{ Education } \\
\hline Primary & $18(14.9)$ \\
\hline Secondary & $57(47.1)$ \\
\hline \multirow[t]{2}{*}{ Tertiary } & $46(38.0)$ \\
\hline & Mean \pm SD \\
\hline Age (years) & $64.77 \pm 9.03$ \\
\hline \multicolumn{2}{|l|}{ Cataract* $^{*}$} \\
\hline NO & $1.64 \pm 0.81$ \\
\hline NC & $2.41 \pm 0.95$ \\
\hline$C$ & $0.88 \pm 1.08$ \\
\hline PSC & $0.37 \pm 0.6$ \\
\hline \multicolumn{2}{|l|}{ BCVA } \\
\hline Study eye & $99.97 \pm 5.88$ \\
\hline Fellow eye & $95.34 \pm 11.45$ \\
\hline \multicolumn{2}{|l|}{ Letter CS (cpd) } \\
\hline 1.2 & $1.81 \pm 0.17$ \\
\hline 2.4 & $1.80 \pm 0.20$ \\
\hline 6 & $1.52 \pm 0.23$ \\
\hline 9.6 & $1.27 \pm 0.28$ \\
\hline $15.15^{*}$ & $0.90 \pm 0.32$ \\
\hline \multicolumn{2}{|l|}{ CSmesopic (cpd) } \\
\hline 1.5 & $1.57 \pm 0.22$ \\
\hline 3 & $1.65 \pm 0.21$ \\
\hline 6 & $1.27 \pm 0.35$ \\
\hline 12 & $0.81 \pm 0.27$ \\
\hline 18 & $0.32 \pm 0.11$ \\
\hline \multicolumn{2}{|l|}{ CSphotopic (cpd) } \\
\hline 1.5 & $1.49 \pm 0.17$ \\
\hline 3 & $1.76 \pm 0.21$ \\
\hline 6 & $1.63 \pm 0.31$ \\
\hline 12 & $1.22 \pm 0.37$ \\
\hline 18 & $0.57 \pm 0.34$ \\
\hline \multicolumn{2}{|l|}{ GDmesopic (cpd) } \\
\hline 1.5 & $0.95 \pm 0.31$ \\
\hline 3 & $1.15 \pm 0.35$ \\
\hline 6 & $0.93 \pm 0.24$ \\
\hline 12 & $0.64 \pm 0.13$ \\
\hline 18 & $0.30 \pm 0.03$ \\
\hline \multicolumn{2}{|c|}{ GDphotopic (cpd) } \\
\hline 1.5 & $1.43 \pm 0.19$ \\
\hline 3 & $1.70 \pm 0.20$ \\
\hline 6 & $1.54 \pm 0.32$ \\
\hline 12 & $1.15 \pm 0.37$ \\
\hline 18 & $0.54 \pm 0.33$ \\
\hline PRT & $15.98 \pm 8.23$ \\
\hline \multicolumn{2}{|l|}{ Reading } \\
\hline RAcuity & $0.10 \pm 0.12$ \\
\hline Mean RS & $154.68 \pm 27.36$ \\
\hline Max RS & $199.79 \pm 33.02$ \\
\hline \multicolumn{2}{|l|}{ SVF } \\
\hline VFQ_Tscore & $88.95 \pm 9.84$ \\
\hline
\end{tabular}

related to MP, controlling for age, sex and cataract grade; these results are reported in the text, separately for each visual function variable. 


\section{Table 1 Continued}

\begin{tabular}{ll}
\hline Variables & $\mathbf{n}(\%)$ \\
\hline $\mathrm{MP}^{*}$ & \\
0.25 & $0.75 \pm 0.25$ \\
0.5 & $0.63 \pm 0.21$ \\
1 & $0.44 \pm 0.17$ \\
1.75 & $0.31 \pm 0.14$
\end{tabular}

Cataracts graded using the Lens Opacities Classification System (LOCS) III. Cataract grades - NO, nuclear opalescence; NC, nuclear colour; C, cortical; PSC, posterior subcapsular cataract.

Data displayed are mean $\pm S D$ for interval data and percentages for categorical data. ${ }^{*} n \neq 121$ for all variables as certain tests/measures could not be obtained. Age, age in years; BCVA, best corrected visual acuity measured with Thompson Test Chart 2000 Xpert and recorded in visual acuity rating (VAR); cpd, cycles per degrees; CS, contrast sensitivity; CSmesopic, contrast sensitivity measured under nighttime conditions (3.0 candela per metre square $\left.\left(\mathrm{cd} / \mathrm{m}^{2}\right)\right)$ using the Functional Vision Analyzer and recorded in logarithm of contrast sensitivity (LogCS) units; CSphotopic, contrast sensitivity measured under daytime conditions $\left(85 \mathrm{~cd} / \mathrm{m}^{2}\right)$ using the Functional Vision Analyzer and recorded in logarithm of contrast sensitivity (LogCS) units; education, highest level of education; GD, glare disability; GDmesopic, glare disability measured under nighttime conditions (28 Lux) using the Functional Vision Analyzer and recorded in logarithm of contrast sensitivity (LogCS) units; GDphotopic, glare disability measured under daytime conditions (135 Lux) using the Functional Vision Analyzer and recorded in logarithm of contrast sensitivity (LogCS) units; LetterCS, letter contrast sensitivity (assessed using Thompson Test Chart 2000 PRO and recorded in logarithm of contrast sensitivity (LogCS) units); maxRS, maximum reading speed; meanRS, mean reading speed (calculated as the average of the reading speed scores recorded for each of the standardised sentences); MP, macular pigment measured at $0.25^{\circ}, 0.5^{\circ}, 1.0^{\circ}$ and $1.75^{\circ}$ eccentricities using customised heterochromatic flicker photometry; n, number of subjects; PRT, photostress recovery time recorded in seconds (reading assessed using the English version of the standardised Radner reading chart); RAcuity, reading acuity (Reading acuity is recorded in logarithm of the reading acuity determination (LogRAD). The formula (logRAD+total number of incorrectly read syllables $\times 0.005$ ) is used to calculate the LogRAD-score.); RS, reading speed (the time taken to read the number of words in a sentence) measured in words per minute $(\mathrm{w} / \mathrm{min})$ with a stop watch for each standardised sentence ( 14 words $\times 60 \mathrm{~s}$ divided by reading time in seconds); SVF, subjective visual function assessed using the National Eye Institute Visual Function Questionnaire-25 (NEI VFQ-25; score range from 0 (worst) to 100 (best)); VFQ_Tscore, NEI VFQ-25 overall vision score.

\section{MP and BCVA}

There is a positive and significant relationship between central MP $\left(0.25^{\circ}\right.$ up to $\left.1^{\circ}\right)$ and BCVA (table 2). In a general linear model controlling for age, sex and cataract grade, there is no statistically significant relationship between MP (at any eccentricity) and BCVA ( $p>0.05$, for all).

\section{MP and letter CS}

MP is not significantly related to letter CS, except for MP at $1^{\circ}$ and letter CS at $1.2 \mathrm{cpd}$ (table 2). In a general linear model controlling for age, sex and cataract grade, letter CS at $1.2 \mathrm{cpd}$ remains positively and significantly related to $\mathrm{MP}$ at $1^{\circ}$ $(\mathrm{p}=0.023$; figure $1 \mathrm{~A})$.

\section{MP and measures of CS using the Functional Vision Analyzer} A range of mesopic CS variables are positively and significantly related to MP (table 2). In a general linear model controlling for age, sex and cataract grade, the following relationships remained statistically significant: (a) the relationship between MP at $0.5^{\circ}$ and mesopic CS at $3 \mathrm{cpd}(\mathrm{p}=0.047)$ and $6 \mathrm{cpd}(\mathrm{p}=0.033)$ (figure 1A); (b) the relationship between MP at $1^{\circ}$ and mesopic CS at $1.5 \mathrm{cpd}(\mathrm{p}=0.033$; figure $1 \mathrm{~A})$. However, $\mathrm{MP}$ at $1.75^{\circ}$ is now positively and significantly related to mesopic CS at $1.5 \mathrm{cpd}(\mathrm{p}=0.041)$ (figure 1A).

For photopic CS and MP, correlations similar to those between MP and mesopic CS are observed (table 2). In a general linear model controlling for age, sex and cataract grade: (a) there is a significant relationship between MP at $0.25^{\circ}$ and photopic CS at $12 \mathrm{cpd}(\mathrm{p}=0.013$; figure $1 \mathrm{~A})$; (b) there is a significant relationship between MP at $0.5^{\circ}$ and photopic CS at $6 \mathrm{cpd}(\mathrm{p}=0.006)$ and $12 \mathrm{cpd}(\mathrm{p}=0.003)$ (figure 1A); (c) there is a significant relationship between MP at $1^{\circ}$ and photopic CS at $6 \mathrm{cpd}(\mathrm{p}=0.039$; figure $1 \mathrm{~A})$ and $12 \mathrm{cpd}(\mathrm{p}=0.025$; figure $1 \mathrm{~B})$. However, MP at $1.75^{\circ}$ is now positively and significantly related to photopic CS at $12 \mathrm{cpd}(\mathrm{p}=0.049$; figure $1 \mathrm{~B})$.

\section{MP and GD}

No significant correlations between MP and mesopic GD are observed (table 2), even after controlling for age, sex and cataract grade $(p>0.05$, for all).

In a general linear model for photopic GD controlling for age, sex and cataract grade, the following relationships remain statistically significant: (a) the relationship between MP at $0.25^{\circ}$ and photopic GD at $6 \mathrm{cpd}(\mathrm{p}=0.006$; figure $1 \mathrm{~B})$; (b) the relationship between MP at $0.5^{\circ}$ and photopic GD at $3 \mathrm{cpd}(\mathrm{p}=0.025)$ and $6 \mathrm{cpd}(\mathrm{p}=0.004)$ (figure 1B); (c) the relationship between MP at $1^{\circ}$ and photopic GD at $3 \mathrm{cpd}(\mathrm{p}=0.030)$ (figure 1B). However, $\mathrm{MP}$ at $1.75^{\circ}$ is now positively and significantly related to photopic GD at $3 \mathrm{cpd}(\mathrm{p}=0.037$ ) (figure $1 \mathrm{~B})$.

\section{MP and reading performance}

Correlations between MP and reading performance are shown in table 2. In a general linear model controlling for age, sex and cataract grade, there is a significant relationship between mean reading speed and MP at eccentricities $1.0^{\circ}(\mathrm{p}=0.046)$ and $1.75^{\circ}(\mathrm{p}=0.034)$ (figure $\left.1 \mathrm{~B}\right)$.

Of note, these reading performance variables are also significantly related to education ( $\mathrm{p}<0.05$, for all). Therefore, we repeated the analyses controlling for age, sex, cataract grade and education. In a general linear model, and after controlling for age, sex, cataract grade and education, only the significant and positive relationship between MP at $1.75^{\circ}$ and mean reading speed persisted $(\mathrm{p}=0.048)$.

\section{MP and subjective visual function}

Correlations between MP and subjective visual function are shown in table 2. In a general linear model controlling for age, sex and cataract grade, MP (at any eccentricity) is not related to subjective visual function $(\mathrm{p}>0.05$, for all).

\section{MP and PRT}

MP (at any eccentricity) is not related to PRT, either in the correlation analyses (table 2) or in the general linear model analyses controlling for age, sex and cataract grade ( $p>0.05$, for all).

\section{DISCUSSION}

This study presents findings on the relationship between MP and visual function in subjects with early AMD who are not yet using supplements (CREST AMD baseline data).

Our main findings show that MP relates to several measures of visual function, even after controlling for age, sex and cataract grade. Indeed, we report that MP is positively associated with a range of CS measures in both mesopic (nighttime) and photopic (daytime) conditions. A possible explanation for the role that MP plays in optimising CS may rest on the visibility hypothesis of MP. This hypothesis posits that MP can enhance detail of a target by the absorption of blue haze. ${ }^{5}$ Blue haze is caused by scattered short-wavelength dominant air light (blue light) that produces a veiling luminance when we view objects at a distance. ${ }^{5} \mathrm{MP}$ accentuates the luminance of an object relative to its background by attenuating this scattered (veiling) shortwavelength visible blue light and, by consequence, extends the 
Table 2 Relationship between MP and visual function using baseline data in the Central Retinal Enrichment Supplementation Trial (CREST) age-related macular degeneration study

\begin{tabular}{|c|c|c|c|c|}
\hline Variables & MP at 0.25 & MP at 0.5 & MP at 1.0 & MP at 1.75 \\
\hline BCVA & $r=0.241, p=0.009$ & $r=0.243, p=0.008$ & $r=0.210, p=0.022$ & $r=0.107, p=0.248$ \\
\hline \multicolumn{5}{|l|}{ Letter CS (cpd) } \\
\hline 1.2 & $r=0.101, p=0.278$ & $r=0.113, p=0.225$ & $r=0.219, p=0.017$ & $r=0.102, p=0.272$ \\
\hline 2.4 & $r=0.094, p=0.310$ & $r=0.116, p=0.210$ & $r=0.171, p=0.064$ & $r=0.020, p=0.829$ \\
\hline 6 & $r=0.166, p=0.073$ & $r=0.157, p=0.089$ & $r=0.153, p=0.098$ & $r=0.016, p=0.859$ \\
\hline 9.6 & $r=0.113, p=0.221$ & $r=0.096, p=0.302$ & $r=0.082, p=0.375$ & $\mathrm{r}=-0.014, \mathrm{p}=0.883$ \\
\hline 15.15 & $r=0.106, p=0.257$ & $r=0.106, p=0.256$ & $r=0.080, p=0.393$ & $r=0.037, p=0.690$ \\
\hline \multicolumn{5}{|l|}{ CSmesopic (cpd) } \\
\hline 1.5 & $r=0.103, p=0.267$ & $r=0.123, p=0.185$ & $r=0.194, p=0.035$ & $r=0.131, p=0.157$ \\
\hline 3 & $r=0.139, p=0.133$ & $r=0.217, p=0.018$ & $r=0.165, p=0.074$ & $r=0.038, p=0.680$ \\
\hline 6 & $r=0.169, p=0.067$ & $r=0.203, p=0.028$ & $r=0.148, p=0.111$ & $r=0.111, p=0.230$ \\
\hline 12 & $r=0.209, p=0.023$ & $r=0.220, p=0.017$ & $r=0.209, p=0.023$ & $r=0.159, p=0.086$ \\
\hline 18 & $\mathrm{r}=0.175, \mathrm{p}=0.058$ & $r=0.170, p=0.066$ & $r=0.071, p=0.447$ & $r=0.228, p=0.013$ \\
\hline \multicolumn{5}{|c|}{ CSphotopic (cpd) } \\
\hline 1.5 & $r=-0.002, p=0.987$ & $r=0.050, p=0.589$ & $r=0.097, p=0.295$ & $r=-0.025, p=0.787$ \\
\hline 3 & $r=0.091, p=0.326$ & $r=0.162, p=0.079$ & $r=0.137, p=0.138$ & $r=0.125, p=0.178$ \\
\hline 6 & $r=0.168, p=0.069$ & $r=0.243, p=0.008$ & $r=0.194, p=0.036$ & $r=0.075, p=0.422$ \\
\hline 12 & $r=0.259, p=0.005$ & $r=0.290, p=0.001$ & $r=0.238, p=0.009$ & $r=0.169, p=0.068$ \\
\hline 18 & $r=0.123, p=0.186$ & $r=0.144, p=0.119$ & $r=0.067, p=0.468$ & $r=0.062, p=0.502$ \\
\hline \multicolumn{5}{|c|}{ GDmesopic (cpd) } \\
\hline 1.5 & $r=0.051, p=0.580$ & $r=0.068, p=0.463$ & $r=0.069, p=0.461$ & $r=0.002, p=0.983$ \\
\hline 3 & $r=0.077, p=0.407$ & $r=0.113, p=0.225$ & $r=0.070, p=0.454$ & $\mathrm{r}=-0.010, \mathrm{p}=0.916$ \\
\hline 6 & $r=0.041, p=0.656$ & $r=0.098, p=0.293$ & $r=0.032, p=0.733$ & $r=-0.096, p=0.303$ \\
\hline 12 & $r=0.029, p=0.758$ & $r=0.060, p=0.520$ & $r=0.067, p=0.474$ & $\mathrm{r}=-0.034, \mathrm{p}=0.713$ \\
\hline 18 & $r=0.047, p=0.611$ & $r=0.080, p=0.390$ & $r=0.042, p=0.654$ & $r=0.031, p=0.740$ \\
\hline \multicolumn{5}{|c|}{ GDphotopic (cpd) } \\
\hline 1.5 & $r=0.051, p=0.580$ & $r=0.083, p=0.374$ & $r=0.081, p=0.382$ & $r=0.061, p=0.515$ \\
\hline 3 & $r=0.164, p=0.076$ & $r=0.256, p=0.005$ & $r=0.231, p=0.012$ & $r=0.153, p=0.098$ \\
\hline 6 & $r=0.233, p=0.011$ & $r=0.242, p=0.008$ & $r=0.186, p=0.043$ & $r=0.116, p=0.211$ \\
\hline 12 & $r=0.222, p=0.016$ & $r=0.225, p=0.014$ & $r=0.164, p=0.077$ & $r=0.085, p=0.359$ \\
\hline 18 & $r=0.166, p=0.073$ & $r=0.180, p=0.051$ & $r=0.070, p=0.450$ & $r=0.069, p=0.458$ \\
\hline PRT & $r=0.073, p=0.433$ & $r=0.153, p=0.097$ & $r=0.068, p=0.464$ & $r=0.001, p=0.990$ \\
\hline \multicolumn{5}{|l|}{ Reading } \\
\hline RAcuity & $r=-0.183, p=0.047$ & $r=-0.187, p=0.042$ & $\mathrm{r}=-0.113, \mathrm{p}=0.152$ & $r=-0.056, p=0.547$ \\
\hline Mean RS & $r=0.177, p=0.056$ & $r=0.170, p=0.065$ & $r=0.222, p=0.016$ & $r=0.132, p=0.153$ \\
\hline Max RS & $r=0.135, p=0.146$ & $r=0.119, p=0.201$ & $r=0.164, p=0.076$ & $r=0.040, p=0.664$ \\
\hline \multicolumn{5}{|l|}{ SVF } \\
\hline VFQ_Tscore & $r=0.188, p=0.042$ & $r=0.132, p=0.155$ & $r=0.124, p=0.181$ & $r=0.025, p=0.787$ \\
\hline
\end{tabular}

Bold typeface signifies statistically significant $p$ value $(p<0.05)$.

BCVA, best corrected visual acuity measured with Thompson Test Chart 2000 Xpert and recorded in visual acuity rating (VAR); cpd, cycles per degrees; CS, contrast sensitivity;

CSmesopic, contrast sensitivity measured under nighttime conditions $\left(3.0\right.$ candela per metre square $\left.\left(\mathrm{cd}^{2} / \mathrm{m}^{2}\right)\right)$ using the Functional Vision Analyzer and recorded in logarithm of contrast sensitivity (LogCS) units; CSphotopic, contrast sensitivity measured under daytime conditions $\left(85 \mathrm{~cd} / \mathrm{m}^{2}\right)$ using the Functional Vision Analyzer and recorded in logarithm of contrast sensitivity (LogCS) units; GD, glare disability; GDmesopic, glare disability measured under nighttime conditions (28 Lux) using the Functional Vision Analyzer and recorded in logarithm of contrast sensitivity (LogCS) units; GDphotopic, glare disability measured under daytime conditions (135 Lux) using the Functional Vision Analyzer and recorded in logarithm of contrast sensitivity (LogCS) units; LetterCS, letter contrast sensitivity assessed using Thompson Test Chart 2000 PRO and recorded in logarithm of contrast sensitivity (LogCS) units; maxRS, maximum reading speed; meanRS, mean reading speed calculated as the average of the reading speed scores recorded for each of the standardised sentences; MP, macular pigment measured at $0.25^{\circ}, 0.5^{\circ}, 1.0^{\circ}$ and $1.75^{\circ}$ eccentricities using customised heterochromatic flicker photometry; $p$, level of statistical significance set at $p<0.05$; PRT, photostress recovery time recorded in seconds (reading assessed using the English version of the standardised Radner reading chart); $r$, Pearson's correlation coefficient; RAcuity, reading acuity (Reading acuity is recorded in logarithm of the reading acuity determination (LogRAD). The formula (logRAD+total number of incorrectly read syllables $\times 0.005$ ) is used to calculate the LogRAD-score.); RS, reading speed (the time taken to read the number of words in a sentence) measured in words per minute (w/min) with a stop watch for each standardised sentence (14 words $\times 60$ s divided by reading time in seconds); SVF, subjective visual function assessed using the National Eye Institute Visual Function Questionnaire-25 (NEI VFQ-25; scores range from 0 (worst) to 100 (best)); VFQ_Tscore, NEI VFQ-25 overall vision score.

visual range. The visibility hypothesis has been tested empirically and is supported by two studies which demonstrate the beneficial effect of MP on simulated blue haze conditions. ${ }^{8} 9$ Furthermore, Hammond and Renzi propose that MP may improve CS by the differential absorption of chromatic edges (ie, the ability of MP to absorb the short-wavelength (blue) component of an isoluminant edge). ${ }^{710} 11$ Of note, we found significant associations between MP and CS at $6 \mathrm{cpd}$, which is the primary outcome measure of the prospective arm of the current study. ${ }^{17}$ The importance of our findings with respect to the relationship between MP and CS rests on the observation that CS has been shown to be an important determinant of quality of life, ${ }^{21}$ which has important implications for the population studied here.

In our study, we also found that reading performance is related to MP levels (table 2). For example, we found that 
A
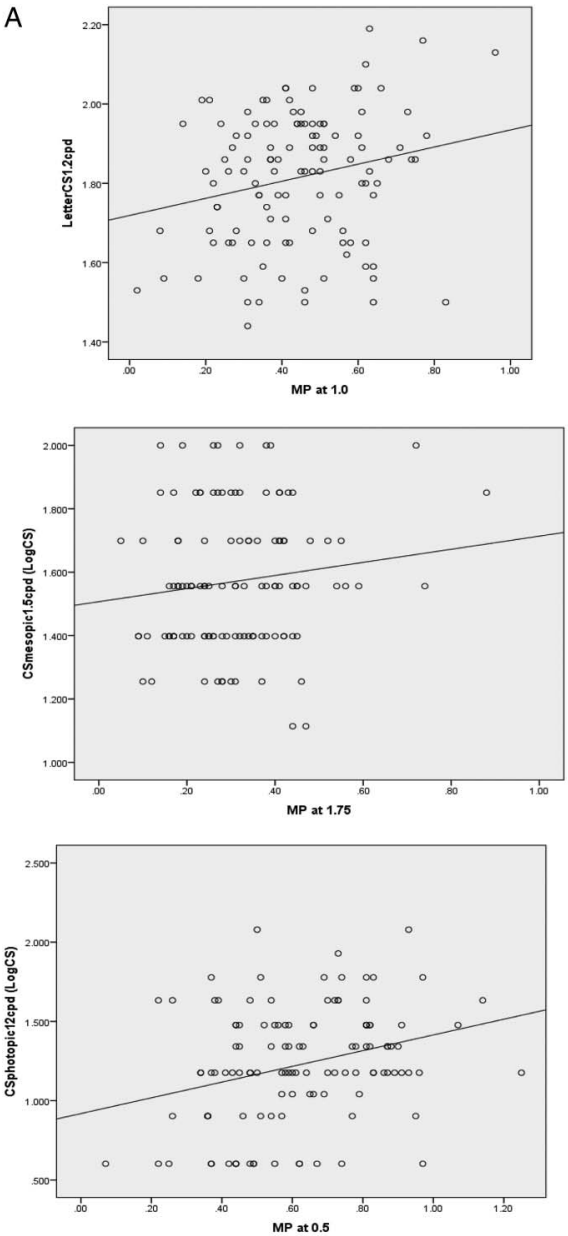
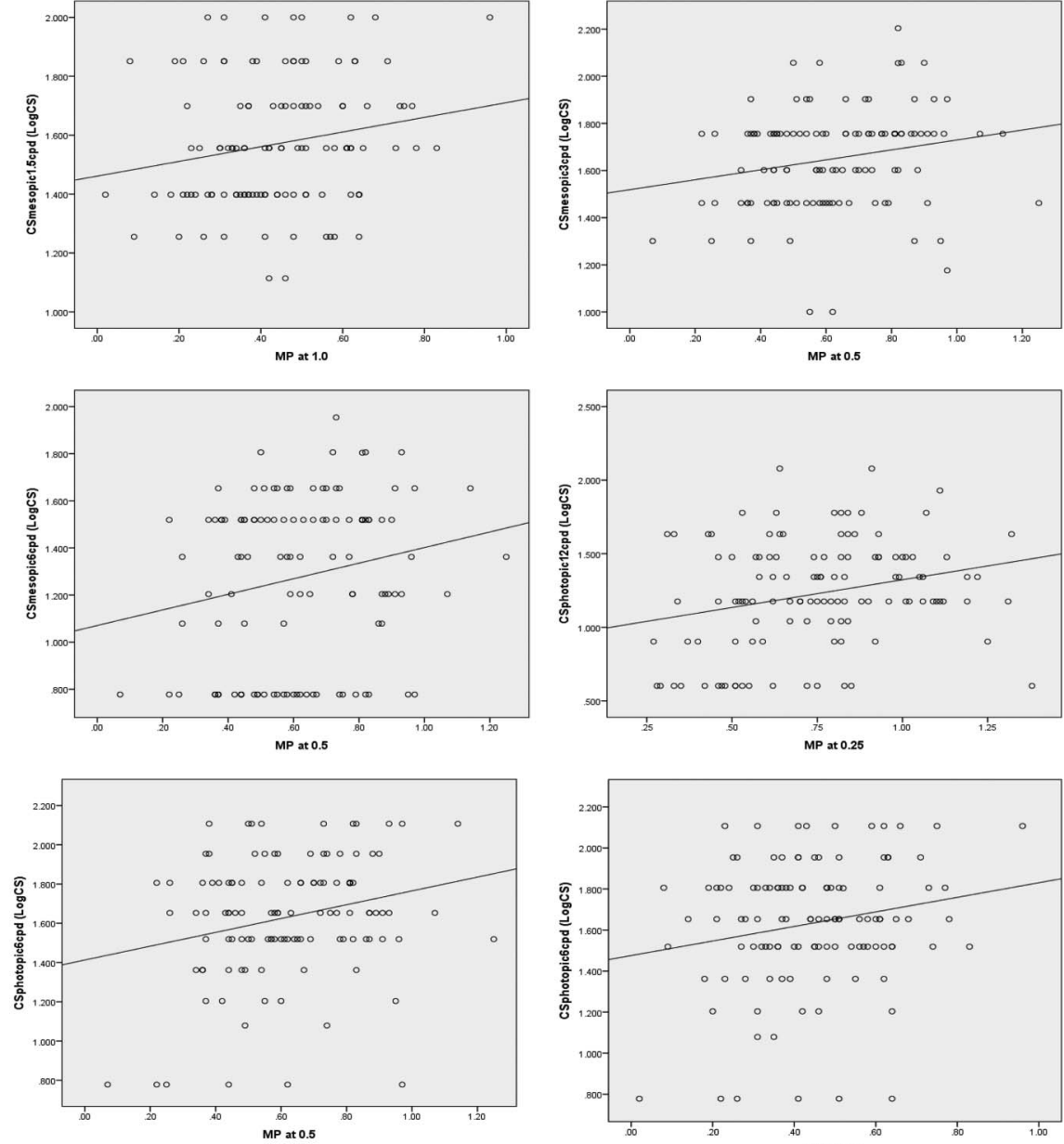

Figure 1 Relationship between measures of macular pigment and visual function. CSmesopic, contrast sensitivity measured under nighttime conditions (3.0 candela per metre square $\left.\left(\mathrm{cd} / \mathrm{m}^{2}\right)\right)$ using the Functional Vision Analyzer and recorded in logarithm of contrast sensitivity (LogCS) units; CSphotopic, contrast sensitivity measured under daytime conditions $\left(85 \mathrm{~cd} / \mathrm{m}^{2}\right)$ using the Functional Vision Analyzer and recorded in LogCS units; GD, glare disability; GDmesopic, GD measured under nighttime conditions (28 Lux) using the Functional Vision Analyzer and recorded in LogCS units; GDphotopic, GD measured under daytime conditions (135 Lux) using the Functional Vision Analyzer and recorded in LogCS units; LetterCS, letter contrast sensitivity.

higher MP at $1^{\circ}$ is associated with better reading speed, even after controlling for age, sex and cataract grade. However, we believe that this finding is not solely attributable to the optical filtration properties of MP at the macula, as it is possible that the role of carotenoids in brain health ${ }^{22}$ and cognition ${ }^{23}$ may have contributed to our finding (and therefore that MP simply represents a biomarker for concentrations of these nutrients in brain). Indeed, this notion is consistent with our finding that education is also related to reading performance in the current study, although it is noteworthy that MP at $1.75^{\circ}$ eccentricity was positively related to mean reading speed even after correction for education. Indeed, and for instance, we know that $\mathrm{L}$ and $\mathrm{Z}$ concentrations at the macula correlate with their respective concentrations in the frontal cortex and cerebellum (brain areas engaged in reading performance) in primates. ${ }^{24}$ It has also been reported that reading speed is positively related to visual processing in persons with AMD. ${ }^{25}$ These carotenoids, and their putative contribution to optimal neural processing and efficiency, ${ }^{26}{ }^{27}$ may contribute positively to reading performance. Reading performance is also a measure of subjects' ability to perform tasks related to near-vision, with important and positive implications for quality of life. ${ }^{28}$
Although the current study did not find any relationship between MP and PRT or mesopic GD, we found a significant and positive relationship between MP and photopic GD at some spatial frequencies (even after controlling for age, sex and cataract grade). Some previous cross-sectional studies have shown that MP is inversely related to GD and PRT, ${ }^{29}{ }^{30}$ and that augmentation of MP results in improvements in GD and PRT, ${ }^{11}{ }_{13}$ whereas others have found no association with GD (or PRT). ${ }^{6}$ These inconsistencies may be attributable to the differences in study design, including stimulus conditions. Of note, glare is an important clinical symptom, and reducing GD would be beneficial for patients with early AMD, especially for driving. ${ }^{31}$

We found that higher central MP (ie, at $0.25^{\circ}, 0.5^{\circ}, 1^{\circ}$ ) is associated with better BCVA in our study group (table 2). However, after controlling for age, sex and cataract grade, there was no significant relationship between MP (at any eccentricity) and BCVA. The acuity hypothesis posits that MP is necessary for optimal visual acuity by reducing the effects of chromatic aberration, ${ }^{3}$ by attenuating the penumbra/blur circle formed as a result of this phenomenon. ${ }^{32}$ However, studies have tested this hypothesis, and the results have been mixed, perhaps, explained at least in part, by differences in study design and outcome 

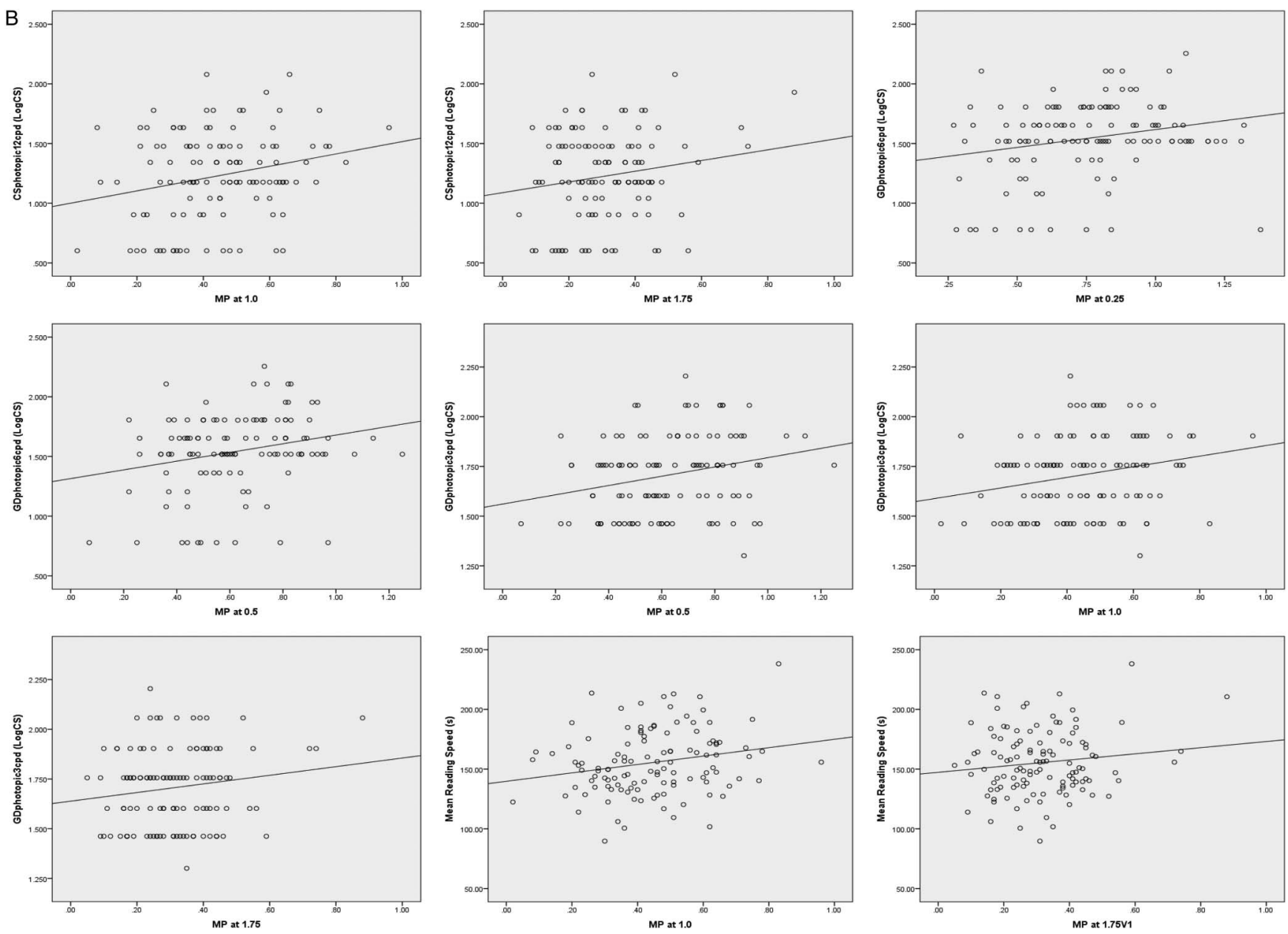

Figure 1 Continued.

measures. ${ }^{6} 33{ }^{34}$ For example, our results are consistent with those of a study by Engles et al, ${ }^{34}$ who assessed resolution acuity and hyperacuity using a customised experimental setup (ie, two solid black lines on either white or yellow background), whereas they are not consistent with those reported by Loughman et al, ${ }^{6}$ who used a methodology similar to the current study, although in normal subjects free of retinal disease.

In this study we performed multiple statistical tests (eg, table 2 presents $p$ values for more than 120 different tests of bivariate correlations). For any one such test, the level of significance is, conventionally, set at 5\%. For correlation analysis, this means that we start with the null hypothesis ('the correlation between the two variables is zero in the population'), then calculate the sample correlation and then reject the null hypothesis if the probability is less than $5 \%$ of getting this sample correlation when the null hypothesis is actually true. For multiple tests, however, the recommendation is sometimes made that the level of significance should be reduced well below 5\%, so as to reduce the probability of a type I error-rejecting null hypotheses which we should accept-arising from the multiplicity of tests. In particular, Bonferroni adjustment is often advocated for multiple tests. This would entail, for the tests reported in table 2, reducing the significance level from 0.05 to about 0.0004 (which is 0.05 divided by 120). Not one of the 27 correlations in table 2 (significant at the 5\% level) would still be reported as significant at this reduced level of significance. We regard this approach as extreme and unwise, however, because it greatly increases the risk of a type II error-accepting null hypotheses which we should reject. This, to us, is the more serious error, failing to report a relationship that does, in fact, exist. In the current study, therefore, we followed our usual practice and used the 5\% level of significance in all statistical analyses, without adjusting for multiple comparisons. It must be conceded, however, that the reported significance for some of our 27 correlations may well, therefore, be spurious.

It must also be conceded that the significant correlations in table 2 are, in general, weak. However, there is remarkable consistency in the direction and location of many of the significant correlations in table 2. In particular, many of the correlations between central MP and CS, when significant at $6 \mathrm{cpd}$, are significant, or nearly so, at $3 \mathrm{cpd}$ and $12 \mathrm{cpd}$ also. Furthermore, all significant correlations between MP and visual function are, without exception, positive-higher $\mathrm{MP}$ is associated with better vision. While the importance of a single correlation of this magnitude should not be exaggerated (and might well be explained as a consequence of multiple tests), we believe that the frequency and consistent directionality of these significant correlations, in our study, do constitute a substantial body of evidence that MP and visual function are positively correlated.

Average central MP (see table 1) may be considered higher than expected in this study, given that subjects were recruited only if they had not previously taken supplements containing the macular carotenoids and, accordingly, all subjects were supplement-naive. The eligibility criteria did not include a threshold for MP at a specific retinal eccentricity. In other words, subjects with a range of MP values were enrolled into 
the study. Of note, MP is augmented either through the diet or by taking nutritional supplements containing the macular carotenoids. Given that subjects in the current study were supplement-naive patients with early AMD, it is more likely that the high average central MP may reflect healthy dietary habits in our study population. Indeed, results from the dietary carotenoid (L/Z) screener used in the current study (see detailed description in Akuffo $e t \quad a l^{17}$ ) show that subjects had, on average, medium dietary intake of $\mathrm{L}$ and $\mathrm{Z}$ (which reflects $3-13 \mathrm{mg} /$ day (data previously reported by Kelly et $\left.a l^{35}\right)$ ). Furthermore, there was a positive and statistically significant correlation $(\mathrm{r}=0.343, \mathrm{p}<0.0005)$ between this diet score and MP at $0.25^{\circ}$-evidence that high MP is indeed associated with better diet in this study.

AMD is a multifactorial disease, which causes degenerative changes at the macula and consequential central vision impairment, thereby adversely affecting normal daily activities (eg, reading, driving, watching television and recognising faces) and, ultimately, leading to an overall loss of social independence and reduced quality of life among sufferers of this condition. ${ }^{36}$ Overall, our results highlight the role of MP in vision-related quality of life, and, by extension, the potential benefits of supplementation with the macular carotenoids for improved quality of life through optimised visual function.

Strengths of this study include: (1) MP was measured using a validated technique at four different retinal eccentricities; (2) the outcome measures for the current study are known and important determinants of quality of life in patients afflicted with AMD. Limitations of this study include its cross-sectional design, and therefore its findings are associative. However, the impact of supplementation with the macular carotenoids on visual function in non-advanced AMD is currently under investigation as part of the prospective arm of the CREST trial, which is designed to investigate whether enrichment of MP impacts on visual function in these subjects. ${ }^{17}$

In conclusion, we report that MP relates positively to visual function in AMD-afflicted eyes of unsupplemented subjects, suggesting that augmentation of MP may enhance vision in patients with this condition.

Acknowledgements The authors thank Professor Elizabeth Johnson from Tufts University, USA, for permission to use the 'L/Z screener' for estimating dietary intake of lutein and zeaxanthin in this study.

Contributors Designed the experiment: KOA, SB and JMN. Conducted the experiment: KOA. Analysed/interpreted data: KOA, JS, SB, JMN and TP. Provided materials: KOA, SB, JMN, JS, TP, IL and LC. Wrote initial draft: KOA. Proofed/revised article: KOA, SB, JMN, TP, JS, IL and LC.

Funding This study was funded by the European Research Council (ERC); reference number: 281096. KOA, LC and JMN were funded by the European Research Council. JMN was also funded by the Howard Foundation, Cambridge, UK. TP and IL were funded by the NIHR BMRC at Moorfields Eye Hospital NHS Foundation Trust and UCL IOO, London, UK.

Competing interests JMN and SB do consultancy work for nutraceutical companies in a personal capacity and as directors of Nutrasight Consultancy Limited.

Patient consent Obtained.

Ethics approval Ethical approval was granted by the Research Ethics Committee of the Waterford Institute of Technology (WIT), Waterford, Ireland, and the Ethics Committee of the European Research Council (ERC).

Provenance and peer review Not commissioned; externally peer reviewed.

Open Access This is an Open Access article distributed in accordance with the Creative Commons Attribution Non Commercial (CC BY-NC 4.0) license, which permits others to distribute, remix, adapt, build upon this work non-commercially, and license their derivative works on different terms, provided the original work is properly cited and the use is non-commercial. See: http://creativecommons.org/ licenses/by-nc/4.0/

\section{REFERENCES}

1 Hammond BR, Wooten BR, Snodderly DM. Individual variations in the spatial profile of human macular pigment. J Opt Soc Am A Opt Image Sci Vis 1997;14:1187-96.

2 Khachik F, Bernstein PS, Garland DL. Identification of lutein and zeaxanthin oxidation products in human and monkey retinas. Invest Ophthalmol Vis Sci 1997:38:1802-11.

3 Schultze M. Ueber den gelben Fleck der Retina, seinen Einfluss auf normales Sehen und auf Farbenblindheit. Cohen \& Sohn, 1866.

4 Stringham JM, Hammond BR Jr. The glare hypothesis of macular pigment function. Optom Vis Sci 2007;84:859-64.

5 Wooten BR, Hammond BR. Macular pigment: influences on visual acuity and visibility. Prog Retin Eye Res 2002;21:225-40.

6 Loughman J, Akkali MC, Beatty S, et al. The relationship between macular pigment and visual performance. Vision Res 2010;50:1249-56.

7 Hammond BR Jr, Fletcher LM, Elliott JG. Glare disability, photostress recovery, and chromatic contrast: relation to macular pigment and serum lutein and zeaxanthin. Invest Ophthalmol Vis Sci 2013;54:476-81.

8 Fletcher LM, Engles M, Hammond BR Jr. Visibility through atmospheric haze and its relation to macular pigment. Optom Vis Sci 2014:91:1089-96.

9 Hammond BR Jr, Wooten BR, Engles M, et al. The influence of filtering by the macular carotenoids on contrast sensitivity measured under simulated blue haze conditions. Vision Res 2012;63:58-62.

10 Renzi LM, Hammond BR. The effect of macular pigment on heterochromatic luminance contrast. Exp Eye Res 2010;91:896-900

11 Hammond BR, Fletcher LM, Roos F, et al. A double-blind, placebo-controlled study on the effects of lutein and zeaxanthin on photostress recovery, glare disability, and chromatic contrast. Invest Ophthalmol Vis Sci 2014:55:8583-9.

12 Sabour-Pickett S, Beatty S, Connolly E, et al. Supplementation with three different macular carotenoid formulations in patients with early age-related macular degeneration. Retina 2014;34:1757-66.

13 Stringham JM, Hammond BR. Macular pigment and visual performance under glare conditions. Optom Vis Sci 2008:85:82-8.

14 Ma L, Yan SF, Huang YM, et al. Effect of lutein and zeaxanthin on macular pigment and visual function in patients with early age-related macular degeneration. Ophthalmology 2012;119:2290-7.

15 Loughman J, Nolan JM, Howard AN, et al. The impact of macular pigment augmentation on visual performance using different carotenoid formulations. Invest Ophthalmol Vis Sci 2012:53:7871-80.

16 Akuffo KO, Nolan JM, Howard AN, et al. Sustained supplementation and monitored response with differing carotenoid formulations in early age-related macular degeneration. Eye (Lond) 2015;29:902-12.

17 Akuffo KO, Beatty S, Stack J, et al. Central Retinal Enrichment Supplementation Trials (CREST): design and methodology of the CREST randomized controlled trials. Ophthalmic Epidemiol 2014;21:111-23.

18 Davis MD, Gangnon RE, Lee LY, et al. The Age-Related Eye Disease Study severity scale for age-related macular degeneration: AREDS Report No. 17. Arch Ophthalmo 2005:123:1484-98.

19 Stringham JM, Hammond BR, Nolan JM, et al. The utility of using customized heterochromatic flicker photometry (CHFP) to measure macular pigment in patients with age-related macular degeneration. Exp Eye Res 2008;87:445-53.

20 Chylack LT Jr, Wolfe JK, Singer DM, et al. The lens opacities classification system III. The Longitudinal Study of Cataract Study Group. Arch Ophthalmol 1993;111:831-6

21 Bansback N, Czoski-Murray C, Carlton J, et al. Determinants of health related quality of life and health state utility in patients with age related macular degeneration: the association of contrast sensitivity and visual acuity. Qual Life Res 2007:16:533-43.

22 Craft NE, Haitema TB, Garnett KM, et al. Carotenoid, tocopherol, and retinol concentrations in elderly human brain. J Nutr Health Aging 2004;8:156-62.

23 Johnson EJ. A possible role for lutein and zeaxanthin in cognitive function in the elderly. Am J Clin Nutr 2012;96:1161S-5S.

24 Vishwanathan R, Neuringer M, Snodderly DM, et al. Macular lutein and zeaxanthin are related to brain lutein and zeaxanthin in primates. Nutr Neurosci 2013;16:21-9.

25 Cheong AM, Legge GE, Lawrence MG, et al. Relationship between slow visual processing and reading speed in people with macular degeneration. Vision Res 2007:47:2943-55.

26 Renzi LM, Bovier ER, Hammond BR Jr. A role for the macular carotenoids in visual motor response. Nutr Neurosci 2013;16:262-8.

27 Bovier ER, Renzi LM, Hammond BR. A double-blind, placebo-controlled study on the effects of lutein and zeaxanthin on neural processing speed and efficiency. PLOS ONE 2014:9:e108178.

28 Hassell JB, Lamoureux EL, Keeffe JE. Impact of age related macular degeneration on quality of life. Br J Ophthalmol 2006:90:593-6.

29 Stringham JM, Garcia PV, Smith PA, et al. Macular pigment and visual performance in glare: benefits for photostress recovery, disability glare, and visual discomfort. Invest Ophthalmol Vis Sci 2011;52:7406-15. 
30 Putnam CM, Bassi CJ. Macular pigment spatial distribution effects on glare disability. J Optom 2015;8:258-65.

31 Mainster MA, Turner PL. Glare's causes, consequences, and clinical challenges after a century of ophthalmic study. Am J Ophthalmol 2012:153:587-93.

32 Reading VM, Weale RA. Macular pigment and chromatic aberration. J Opt Soc Am A 1974:64:231-4.

33 Puell MC, Palomo-Alvarez C, Barrio AR, et al. Relationship between macular pigment and visual acuity in eyes with early age-related macular degeneration. Acta Ophthalmol 2013;91:e298-303.
34 Engles M, Wooten B, Hammond B. Macular pigment: a test of the acuity hypothesis. Invest Ophthalmol Vis Sci 2007;48:2922-31.

35 Kelly D, Coen RF, Akuffo KO, et al. Cognitive function and its relationship with macular pigment optical density and serum concentrations of its constituent carotenoids. J Alzheimers Dis 2015; 48:261-77

36 Slakter JS, Stur M. Quality of life in patients with age-related macular degeneration: impact of the condition and benefits of treatment. Surv Ophthalmol 2005;50:263-73. 